\title{
Cost-effective scheduling analysis through discrete event simulation for distributed
} systems

\begin{abstract}
Large computing systems where globally distributed can be best characterized by their dynamic nature particularly in terms of resource provisioning and scheduling. Users of the systems normally aim to maximize their own interest when consuming the shared resources. Apart from that, the processing requirements that submitted by the systems' users are diverse in their properties (e.g., size, priority). This condition makes the resources in distributed system overwhelmed by heterogeneity of task to be processed; that leads to fluctuation in resource availability. There are researchers' proposed scheduling algorithms and evaluated through simulation system in order to improve resource availability. It is because the simulation system is able to save cost rather than real test bed experimental. In response to this, we proposed priority-based scheduling algorithm for improving resource availability that developed using discrete-event simulation approach. We defined several events in the simulation to represent various execution statuses that used to monitor resource state in the distributed systems. Our simulation system successfully gives better performance in terms of waiting time compared than other works that also used simulation as their experimental platform.
\end{abstract}

Keyword: Discrete event simulation; Task scheduling; Cost-effective computing; Distributed systems; Heterogeneous resources 